\title{
An Experimental Analysis on EMG Artifact Removal Methods from EEG Signal Records
}

\author{
Sagar S. Motdhare ${ }^{1}$, Dr. Garima Mathur ${ }^{2}$ \\ ${ }^{1}$ Research Scholar, EEE Department, Poornima University, Jaipur, India \\ ${ }^{2}$ Professor, EEE Department, Poornima University, Jaipur, India \\ 1sagar_motdhare@yahoo.com, \\ ${ }^{2}$ drg.mathur@poornima.edu.in
}

\author{
Article Info \\ Page Number: $72-78$ \\ Publication Issue:
}

Vol 71 No. 1 (2022)

\section{Article History}

Article Received: 18 November 2021

Revised: 01 December 2021

Accepted: 15 December 2021

Publication: 27 January 2022

\begin{abstract}
Electromyography is the measurement of the muscle action prospective (MUAP) in many muscle tissue over time and space (EMG). In real time measurements, EMG signals will damage electromyography data, making effective investigation and elucidation of EEG signals difficult. A crucial step is to eliminate distortions of EMG from EEG records. Singular Spectrum Analysis and Multimodal Empirical Mode Decomposition are two new methods for reducing EEG distortions. Using Independent Component Analysis and the Wavelet Method together, for example, some researchers supplied two approaches and then exploited their respective benefits to further eliminate artifacts without hurting the EEG data. New approaches for eliminating muscle artefacts from EEG are studied in this research. Signal transformations, filtering algorithms as well as blind source separation are among the fundamental techniques examined.
\end{abstract}

Keywords: - EEG (Electroencephalography), EMG (Electromyography), Artifacts, Artifact removal.

\section{INTRODUCTION}

Electroencephalography (EEG) records electrical impulses generated by non-brain activities as well as brain activity. Electrooculography, Electro-myography, electro-cardiography in addition to power line intervention are all examples of significant abnormalities. Artefacts have a significant impact on overall EEG analysis, eventually leading to the loss of critical data. As a result, artefact removal is one of the most important pre-processing techniques in the deployment of brain cognition, which is significant for neuroscience investigations and therapy evaluation. [1]

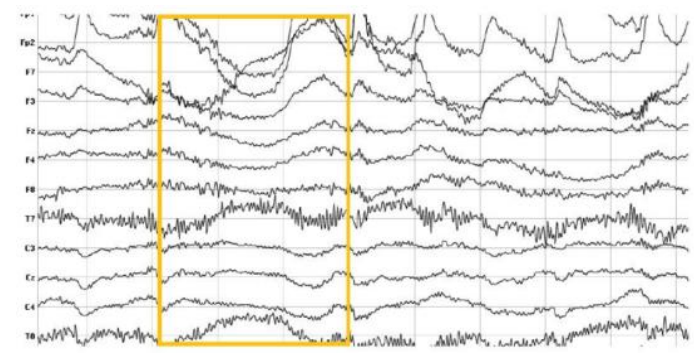

Fig. 1: EMG artefact generated by subjects moving their heads with a swift motion. [1] 
https://doi.org/10.17762/msea.v71i1.43

One of the most prevalent strategies for eliminating artefacts in EEG recordings is regression. In 1970, Hillyard et al. published the first time domain regression method for removing EEG distortions [3]. Whitton then refined the frequency domain regression method by integrating it with EEG revealing software [4]. Adaptive filtering was applied by P He et al. to reduce ocular artefacts in EEG, providing simplified, steady and quick settling. [1][5].

The introduction of BSS facilitated the development of artefact extraction techniques such as PCA, ICA and CCA. Berg and Scherg were the first to propose PCA as a method for reducing eye component artefacts, and it was proven to be superior than regression and the dipole methodology [6]. In 1996, Makieg and colleagues were among the first to use ICA to investigate standard EEG and EPR [7]. Clercq, W.D., et al. presented CCA to get rid of EMG artefacts on or after EEG for the first time in 2006.[8]

Furthermore, signal decomposition algorithms for removing EEG aberrations have improved with time, with Kumar, One example is S.P.'s use of a Wavelet Transform to remove ocular aberrations from an EEG signal in 2008 [9]. BSS was used to remove artefacts from EEG data using signal decomposition methods. To minimise muscle artefacts in wide EEG, Wavelet with PCA was offered by Kevric and Subasi, however Chen, X. et al. recommended EEMD-CCA. [1, 10, 11, 12].

Signal transforms such as wavelet, Empirical Mode Decomposition, and Multivariate Empirical Mode Decomposition are discussed in this paper. The creation and benefits of two types of filters, the Butterworth analogue filter and the adaptive filter, are also thoroughly investigated. The benefits and drawbacks of BSS are also discussed. At last, fresh approaches like single spectrum analysis and a mechanism to integrate ICA with wavelet have been introduced in recent years.

\section{MethodS}

\section{A. Signal Transforms}

(i) Wavelet Transform (WT) : The Wavelet transform [9] is a variant of the Fourier transform that provides a shifting frequency "time-frequency" window. The wavelet transform refines the signal step by step at low frequencies, eventually achieving time and frequency subdivision. It could also adapt on the fly to time-frequency signal analysis requirements, allowing it to concentrate on any component of the signal.

Despite the fact that the frequency bands of EEG, ECG artefact, and EOG artefact overlap, the wavelet transform requires semi frequency regions of EEG signal and artefacts. As a result, new preprocessing methods commonly combine the wavelet transform with current noise reduction algorithms.

(ii) Empirical Mode Decomposition (EMD) : EMD divides data depending on the data's time scale features rather than a specified primary function, unlike the wavelet transform [12]. As a result, it has a high signal ratio and can be used to assess nonstationary and non-linear signal sequences [13]. The goal of EMD is to find all Internal Oscillatory Modes in a signal using only a signal-specific time scale. The typical time frame and the idea of the IMF are both familiar and estimated in this technique. The EMD method's noise sensitivity, on the other hand, makes mode combining more difficult $[1,14]$.

MEMD is an improved EMD method that analyses inherent modes in numerous channels at the identical time. As a result, MEMD can reduce artefacts (especially broadband muscle artefacts) more efficiently and precisely [15]. Chaolin Teng et al. developed an EMG artefact elimination technique based on M-EMD [16], which split the EEG signal into multiple multivariable eigen-mode functions (MIMFs) of dissimilar incidence bands. The EMG artefact-containing MIMFs were then eliminated, and the remaining MIMFs were utilised to reconstruct the cleaner EEG signal. According to the findings, the SNR of EEG signals was dramatically improved, and the mean square error was significantly reduced. [1] The EMG artefact-containing MIMFs were then eliminated and the residual MIMFs were employed to recreate the uncontaminated EEG signal. According to the findings, the SNR ratio of EEG signals was dramatically enhanced and the MSE was significantly reduced. [1]

\section{B. Filtering Methods}

(i) Butterworth Analog Filter: The Butterworth filter, a category of electrical filter, was created by a Stephen Butterworth in the year 1930. Butterworth filters provide a smooth frequency response curve in the pass band to the greatest extent practicable. To decrease EMG distortions in clinical EEG recordings, John S. Barlow (1984) presented a 4-pole Butterworth analogue filter [17]. Four-pole Butterworth filtering was paired with an earlier variable electrical filter to create prototype filters. Several aspects were then employed for each channel on the assembly board to create a 20-channel device with varied cut-off frequencies. The researchers discovered that by using a $12.5 \mathrm{~Hz}$ cut-off frequency, the filter was able to remove EMG artefacts while minimising 
https://doi.org/10.17762/msea.v71i1.43

EEG signal distortion. The Butterworth analogue filter has the advantage of being able to function continuously on-line, which is needed for regular EEG use. Nonetheless, the EEG and EMG frequency bands overlapped, restricting its applicability. [1]

(ii) Adaptive Filter : Adaptive filtering works by iteratively adjusting the weights in the primary input using an optimization technique to measure the degree of artifactual contamination, and then removing it from EEG signals with artefacts. Figure 2 illustrates adaptive filtering in operation. A combination of clean and pure EEG data, and also an artefact source, makes up the major input.

Jyh-Shing ANFIS (Adaptive Network-based Fuzzy Inference System), developed by Roger Jang, has proven to be a gamechanging technology in recent years. It uses a hybrid methodology that combines the back propagation technique and the least square method [18] to alter the premise and conclusion parameters. This technique was used by C. Kezi Selva Vijilal et al. [19] to remove EEG artefacts such as EOG, EMG, and ECG. While ANFIS can respond quickly and deal with ambiguous and confusing situations, its filtering results should be improved.

Jing $\mathrm{Hu}$ created an adaptive FL-BPNN (Functional Link-Back Propagation Neural Network) filter to alter the parameters of fuzzy rules. [20]. The experimental findings suggest that this filter outperforms the ANFIS filter in terms of performance (MSE is used as the performance assessment index). They improved their technology a year later by creating a new adaptive filter that incorporates FLNN (Functional Link Neural Network) and ANFIS [21]. In terms of MSE and SNR, they compared their technique to the filters outlined above. FLNN-ANFIS has a clear advantage based on the experimental data. [1]

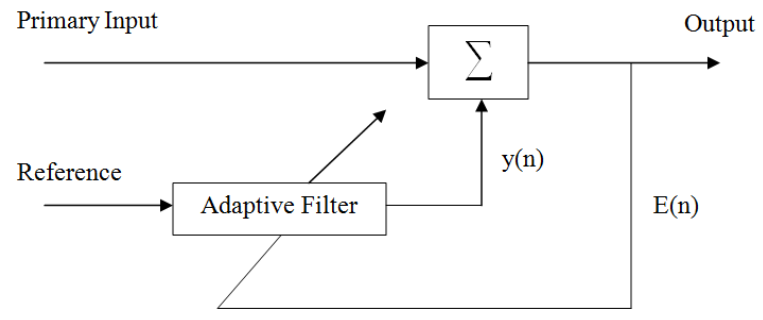

Fig. 2: Functional Block illustration of an Adaptive Filter System [1,22]

\section{Blind Source Separation}

(i) Principal Component Analysis (PCA): PCA converts a set of possibly correlated variables into a set of completely uncorrelated variables via orthogonal transformation. The primary components are the variables that have been modified.'

Berg and Scherg [6] were the first to use PCA to remove eye component artefacts while eliminating topography distortion. The basic components of eye movement and blink artefacts were isolated using an EEG signal. According to Berg and Casarotto's [23] findings, PCA improves the regression and dipole methods.

One or more of PCA's flaws is that satisfying the criterion that artefacts be unrelated to EEG data is challenging. PCA also fails to properly isolate the interference when the drift potential mimics EEG data[1,22].

(ii) Independent Component Analysis (ICA): ICA is a typical BSS technique for separating multiple signals into additive components, based on the supposition that the sub apparatus are non-gaussian signals that look statistically independent of one another. To achieve the goal of noise reduction, ICA removes undesired artefacts (ICs) and reconstructs a clean EEG signal.

In 1996, Makieg et al. used ICA for routine EEG and EPR analysis [7], while Vigaro et al. used ICA to eliminate artefacts from EEG and determine if they were artefacts by examining ICA independent components and their depiction on the electroencephalogram [24, 25]. Jung et al. improved the method in 2000 by applying it to three groups of empirical observations and reporting on the results using PCA and regression methods [26]. In 2003, Romero et al. looked into the effect of ICA on decreasing artefacts at different periods of sleep and revealed that the simultaneous effectiveness of EEG and EOG had only a minor impact on ICA noise reduction. [27]. Joyce et al. introduced a method for automatically extracting and removing eye movement artefacts after ICA analysis in 2004 [28], with results comparable to manual removal. Hybrid techniques based on ICA for automated artefact removal have recently emerged. To automatically suppress ocular artefacts, Raofen Wang et al. employed ICA and fuzzy C-Means clustering algorithms [29]. All of the criteria used to characterise the artefacts and EEG components showed accuracy rates above 99 percent in the experiments. Frlich and Dowding [30] examined the ability of five widely used ICAbased oscillatory activity extraction strategies. The significance of proper high-pass filtering has been established. 
https://doi.org/10.17762/msea.v71i1.43

The advantage of ICA is that it can approximate the non-Gaussian input signals correctly and variably. Whether the signal source is Gaussian or non-Gaussian, however, is a point of contention. Furthermore, biological signal acquisition is not always linear and instantaneous. Two ICA research directions are how to cope with nonlinear convolution signals and how to automatically exploit ICA to reduce artefacts [1,31].

(iii) Canonical Correlation Analysis (CCA): CCA isolates the components from the uncorrelated sources by extracting two sample comprehensive variables from the two sets of variables. To indicate the general association between the two categories of indicators, there is a correlation between the two extensive variables.

The approach was initially used to remove EMG artefacts from EEG by W.D. Clercq and coworkers [8]. The results of this method in medical practise were published by Vergult et al. [31] to improve the interpretation of ictal scalp EEG. BSS-CCA improved seizure localization sensitivity from $62 \%$ to $80 \%$ and eradicated most muscle artefact contamination in ictal EEGs, which is a significant improvement.

CCA, unlike ICA, uses second-order statistics, which requires a faster computation time. Furthermore, while the ICA technique is adequate for removing ocular artefacts, the separation of brain and muscle activities causes true brain activity to be obstructed while EMG artefacts are removed. As a result, when it comes to eliminating muscle artefacts, the CCA approach surpasses the ICA algorithm. [1]

\section{New Approaches}

(i) Singular Spectrum Analysis (SSA) : Singular spectrum analysis, a subspace-based technique for eliminating muscle aberrations from single channel EEG data [33] was projected by AK Maddirala and RA Shaik.

After the single channel signal was transformed into a multi-channel signal, singular value decomposition was used to create orthogonal principal components from the covariance matrix of multi-channel data. An arbitrary cut-off (0.275) was used to find these eigenvectors, and the related subspace of EEG signals was established. After locating the subspace, the multichannel data was merely pushed into it and then back embedded to recover the EEG signal.

As per the findings, EMG artefacts may be effectively eliminated without causing ictal activity to suffer.

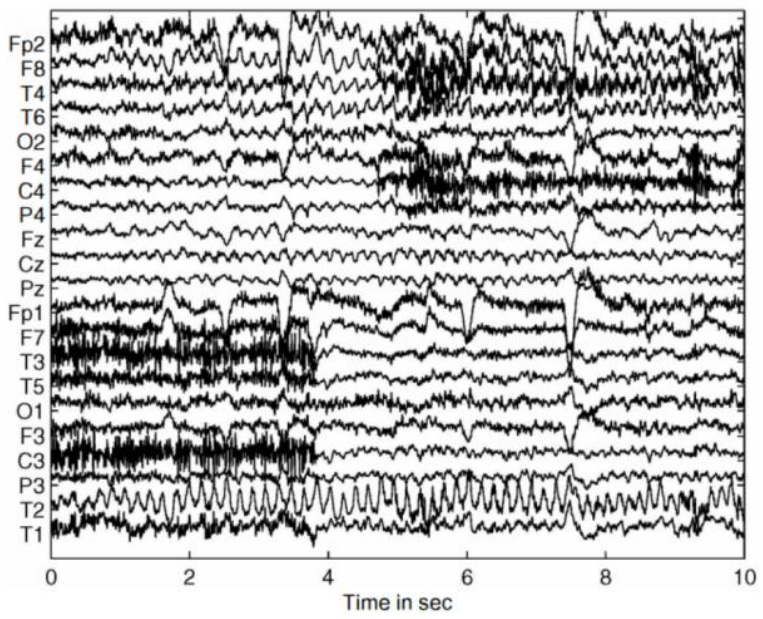

Fig. 3: 10 Sec of 21 Channel EEG Recording [1,32] 


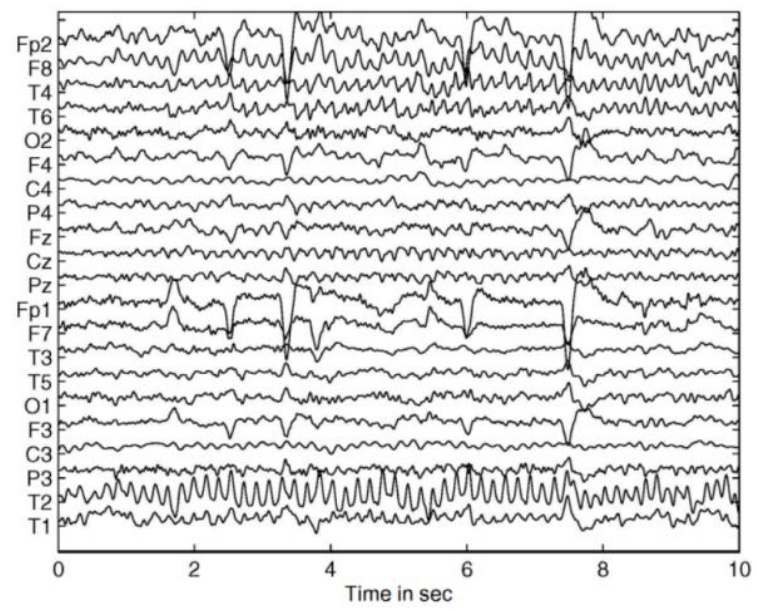

Fig. 4: 10 Sec of 21 Channel EEG Recording after applying the planned scheme [1,32]

(ii) ICA and Wavelet Method: A novel noise reduction strategy based on a blend of ICA and WT is presented to overcome the flaws of ICA and WTs. [1]

Akhtar et al. designed a methodology based on ICA and wavelet de-noising to dynamically eliminate artefacts from multichannel EEG signals (WD). From the given EEG data, ICA was used to extract artifact-only independent components (ICs), and then wavelet de-noising was used to remove any cerebral activity from the artefacts ICs. The major benefit of this method is that it requires less time to compute because all ICs do not need to be identified.

R Kashid and KP Paradeshi used this technique to remove EMG distortions from 16-channel EEG data in 2020. SWT Wavelet decomposition was created using Symlet wavelet and Hard thresholding. EMG artefacts such as teeth clenching, jaw clenching, and forehead movement were reduced using this method. [1]

\section{CONCLUSIONS}

Signal transform, filtering, BSS, and other modern technologies are all addressed above as methods of removing EMG from EEG signals.

The removal of EMG signal interference from EEG is far more difficult than the removal of ECG, EOG, and other artefacts. The main reason for this is that EMG artefact has large amplitude, a broad range, and a physiological pattern that renders numerous common artefact removal techniques ineffective. Because the presence of artefacts has a significant brunt on later EEG investigation and can result in the loss of critical information, removing EMG artefacts is a vital action.

Amid these methods, SSA (Singular Spectrum Analysis) stands out because it effectively reduces muscular artefacts while preserving ictal activity. The ICA and wavelet techniques can remove several EMG aberrations from raw EEG signals such as teeth clenching, clenching of jaws and movement of forehead.

When it comes to removing artefacts, a one-size-fits-all approach rarely works. A successful removal impact may only be achieved by combining multiple ways and allowing full exposure to the advantages of each section.

\section{REFERENCES}

[1] Yu, Mohan. "Removal Methods of EMG Artifacts from EEG Signals." Journal of Physics: Conference Series, vol. 1920, no. 1, 2021, p. 012076., https://doi.org/10.1088/1742-6596/1920/1/012076.

[2] Information from "https://my.oschina.net/u/989000/blog/4557890," 2020.

[3] S. A. Hillyard and R. J. Galambos, "Eye movement artifact in the CNV," Electroencephalography \& Clinical Neurophysiology, vol. 28, no. 2, pp. 173-182, 1970. 
Mathematical Statistician and Engineering Applications

ISSN: 2094-0343

2326-9865

https://doi.org/10.17762/msea.v71i1.43

[4] J. L. Whitton, F. Lue, and H. J. Moldofsky, "A spectral method for removing eye movement artifacts from the EEG," Electroencephalography \& Clinical Neurophysiology, vol. 44, no. 6, pp. 735- 741, 1978.

[5] P. He, G. Wilson, and C. J. Russell, "Removal of ocular artifacts from electro-encephalogram by adaptive filtering," Medical and Biological Engineering and Computing, vol. 42, no. 3, pp. 407-412, 2004.

[6] P. Berg, M. J. C. p. Scherg, D. G. f. r. M. P. physiological measurement : an official journal of the Hospital Physicists' Association, and t. E. F. o. O. f. M. Physics, "Dipole modelling of eye activity and its application to the removal of eye artefacts from the EEG and MEG," Clinical physics physiological measurement, vol. 12 Suppl A, no. A, p. 49, 1991.

[7] T. P. Jung, S. Makeig, A. J. Bell, and T. J. Sejnowski, "Independent Component Analysis of Electroencephalographic and Event-Related Potential Data," Central Auditory Processing and Neural Modeling, 1998.

[8] W. D. Clercq, A. Vergult, B. Vanrumste, W. Van Paesschen, and S. J. Van Huffel, "Canonical correlation analysis applied to remove muscle artifacts from the electroencephalogram," IEEE Transactions on Biomedical Engineering, vol. 53, no. 12, pp. 2583-2587, 2006.

[9] P. S. Kumar, R. Arumuganathan, K. Sivakumar, and C. J. Vimal, "Removal of ocular artifacts in the EEG through wavelet transform without using an EOG reference channel," Int.j.open Probl.comput.math, vol. 1, no. 3, pp. 188-200, 2008.

[10]J. Kevric and A. J. Subasi, "The Effect of Multiscale PCA De-noising in Epileptic Seizure Detection," Journal of Medical Systems, vol. 38, no. 10, p. 131, 2014.

[11]X. Chen, Q. Chen, Y. Zhang, and Z. J. J. Wang, "A Novel EEMD-CCA Approach to Removing Muscle Artifacts for Pervasive EEG," IEEE Sensors Journal, pp. 1-1, 2018.

[12]B. Yang, T. Zhang, Y. Zhang, W. Liu, J. Wang, and K. J. Duan, "Removal of Electrooculogram Artifacts from Electroencephalogram Using Canonical Correlation Analysis with Ensemble Empirical Mode Decomposition," Cognitive Computation, 2017.

[13]N. E. Huang et al., "The empirical mode decomposition and the Hilbert spectrum for nonlinear and non-stationary time series analysis," Proceedings Mathematical \& $\quad$ Physical Engineering Sciences, vol. 454, no. 1971, pp. 903-995, 1998.

[14]X. Xu, C. Xun, and Z. J. E. L. Yu, "Removal of Muscle Artifacts from Few-Channel EEG Recordings Based on Multivariate Empirical Mode Decomposition and Independent Vector Analysis," Electronics Letters, vol. 54, no. 14, 2018.

[15]Chen et al., "The Use of Multivariate EMD and CCA for Denoising Muscle Artifacts From FewChannel EEG Recordings," IEEE Transactions on Instrumentation and Measurement, vol. 67, no. 2, pp. 359-370, 2018.

[16]Y. Z. Chaolin Teng, Gang Wang, "The removal of EMG artifact from EEG signals by the Multivariate Empirical Mode Decomposition," IEEE 2014.

[17]J. S. J. Barlow, "EMG artifact minimization during clinical EEG recordings by special analog filtering," Electroencephalogr Clin Neurophysiol, vol. 58, no. 2, pp. 161-174, 1984.

[18]J.-S. R. Jang, "ANFIS : Adap tive-Ne twork-Based Fuzzy Inference System " IEEE TRANSACTIONS ON SYSTEMS, MAN, AND CYBERNETICS, vol. VOL. 23, NO. 3, 1993.

[19]C. V. P. K. S. J. V. Ewards, "Artifacts Removal in EEG Signal using Adaptive Neuro Fuzzy Inference System," IEEE, vol. Feb. 22-24, pp. 589-591., 2007.

[20] J. H. C. W. M. W. Y. Du, "An Algorithm to Remove Artifacts from EEG Based on Adaptive FLBPNN Filter," Proceedings of the 32nd Chinese Control Conference, vol. July 26-28, 2013.

[21]J. Hu, C. S. Wang, M. Wu, Y. X. Du, Y. He, and J. J. She, "Removal of EOG and EMG artifacts from EEG using combination of functional link neural network and adaptive neural fuzzy inference system," Neurocomputing, vol. 151, no. mar.3pt.1, pp. 278-287, 2014.

[22]"Removal of Artifacts from EEG Signals: A Review."

[23]S. Casarotto, A. M. Bianchi, S. Cerutti, and G. A. J. Chiarenza, "Principal component analysis for reduction of ocular artefacts in event-related potentials of normal and dyslexic children," Clinical Neurophysiology, vol. 115, no. 3, pp. 609619, 2004.

[24]R. N. J. Vigário, "Extraction of ocular artefacts from EEG using independent component analysis," Electroencephalography \& Clinical Neurophysiology, vol. 103 no. 3, pp. 395-404, 1997.

[25]D. R. Vigário, M. J. Särelä, D. V. Jousmäki, D. M. Hämäläinen, and P. E. J. Oja, "Independent component approach to the analysis of EEG and MEG recordings," IEEE Trans Biomed Eng, vol. 47, no. 5, pp. 589-593, 2000. 
[26]T. P. Jung, S. Makeig, M. Westerfield, J. Townsend, and T. J. J. Sejnowski, "Removal of eye activity artifacts from visual event-related potentials in and clinical subjects," Clinical Neurophysiology Official Journal of the International Federation of Clinical Neurophysiology, vol. 111, no. 10, pp. 1745$1758,2000$.

[27]S. Romero, M. A. Mananas, S. Clos, S. Gimenez, and M. J. Barbanoj, "Reduction of EEG artifacts by ICA in different sleep stages," in International Conference of the IEEE Engineering in Medicine \& Biology Society, 2004.

[28]C. A. Joyce, I. F. Gorodnitsky, and M. J. Kutas, "Automatic removal of eye movement and blink artifacts from EEG data using blind component separation," Psychophysiology, vol. 41, no. 2, pp. 313-325, 2010.

[29]R. W. J. Z. X. Wang, "Automatic Ocular Artifact Suppression From Human Operator's EEG Based on a Combination Of Independent Component Analysis and Fuzzy C-Means Clustering Techniques," Proceedings of the 30nd Chinese Control Conference vol. July 22-24, 2011.

[30]L. Frlich and I. J. Dowding, "Removal of muscular artifacts in EEG signals: a comparison of linear decomposition methods," Brain Informatics, vol. 5, no. 1, pp. 13-22, 2018.

[31]Y. L. Xiaoyan Du, Y. zhu, and Q. Ren, "Removal of Artifacts from EEG SignaI," Journal of Biomedical Engineering, vol. 25, no. 2, 2008.

[32]A. K. Maddirala and R. A. Shaik, "Removal of EMG artifacts from single channel EEG signal using singular spectrum analysis," in 2015 IEEE International Circuits and Systems Symposium (ICSyS), 2016. 